\title{
MODELO DE SUPERVISIÓN BASADO EN EL RIESGO PARA INSTITUCIONES PRESTADORAS DE SERVICIOS DE SALUD COMO HERRAMIENTA PARA LA PROTECCION DE LOS DERECHOS EN SALUD EN PERÚ
}

\author{
Vicente A. Benites-Zapata ${ }^{1,2, a, b}$, Héctor A. Saravia-Chong ${ }^{3, c}$, Edward Mezones-Holguin 1,4,a,d, Allen J. Aquije-Díaz ${ }^{3, a, e}$, \\ José Villegas-Ortega ${ }^{1, \mathrm{f,9}}$, Gustavo Rossel-de-Almeida ${ }^{5, \mathrm{a}, \mathrm{h}}$, Carlos Acosta-Saal ${ }^{3, \mathrm{a}}$, Flor Philipps-Cuba ${ }^{6,7, a, \mathrm{i}}$
}

\begin{abstract}
RESUMEN
Objetivos. Describir el modelo de supervisión de las Instituciones Prestadoras de Servicios de Salud (IPRESS) de la Superintendencia Nacional de Salud (SUSALUD) y evaluar los factores asociados al porcentaje de cumplimiento normativo ajustado a riesgo (\%CNAR) en las IPRESS del Sistema de Salud Peruano (SSP). Materiales y Métodos. Se realizó un estudio de caso sobre el desarrollo e implementación de un modelo de supervisión ajustado a riesgo basado en la norma ISO 31000-2009. Con la IPRESS como unidad de análisis, se calcularon los \%CNAR (un puntaje continuo entre 0 a 100) de las supervisiones integrales (SI) y supervisiones selectivas efectuadas durante los años 2013 al 2015. Un mayor \%CNAR implica un menor riesgo operacional. Se estimaron coeficientes $\beta$ con IC95\% mediante modelos lineales generalizados para valorar la asociación entre el \%CNAR (variable de respuesta) y el subsector, la región, el nivel de complejidad y el año de supervisión (variables de exposición). Resultados. Se ejecutaron 1444 supervisiones. En las SI, solo la Seguridad Social en Salud (ESSALUD) tuvo mayor \%CNAR que los centros privados $[(\beta=7,7 \% ;$ IC $95 \%(3,5$ a 11,9)]. Las IPRESS de la Costa $[\beta=-5,2 ;$ IC95\% $(-9,4$ a $-1,0)]$, Sierra $[\beta=-12,5 ;$ IC95\% $(-16,7$ a $-8,3)]$ y Selva $[\beta=-12,6 ;$ IC95\% $(-17,7$ a $-7,6)]$ tuvieron menor \%CNAR que aquellas ubicadas en Lima Metropolitana. El \%CNAR fue superior en el año 2015 [ $\beta=10,8$ IC95\%(6,4 a 15,3)] en relación al año 2013. Conclusiones. El \%CNAR difiere por subsector, región y año de supervisión. En las SI las IPRESS supervisadas en ESSALUD, Lima Metropolitana y el año 2015, tuvieron mejores puntuaciones. Se sugiere la puesta en marcha de acciones orientadas a mejorar el \%CNAR con el propósito de favorecer el ejercicio de los derechos en salud en el SSP.
\end{abstract}

Palabras clave: Gestión de riesgos; Centros de salud; Derecho a la salud; Servicios de salud; Perú (fuente: DECS BIREME).

\section{A RISK-BASED MONITORING MODEL FOR HEALTH CARE SERVICE INSTITUTIONS AS A TOOL TO PROTECT HEALTH RIGHTS IN PERU}

\begin{abstract}
Objectives. To describe the monitoring model of the Health Care Service Institutions (HCSI) of the National Health Authority (NHA) and assess the factors associated with risk-adjusted normative compliance (\%RANC) within the Peruvian Health System (PHS). Materials and Methods. We carried out a case study of the experience of the NHA in the development and implementation of a monitoring program based on the ISO $31000-2009$. With HCSI as the units of analysis, we calculated the \%RANC (a score in continuous scale ranging from 0 to 100) for comprehensive monitoring (CM) and for specific evaluations made from 2013 to 2015. A higher score in the \%RANC means lower operational risk. Also, slope coefficients $(\beta)$ and their $95 \%$ confidence intervals $(95 \% \mathrm{Cl})$ were estimated using generalized linear models to estimate the association between \%RANC as outcome, and health subsector, region, level of care and year, as explanatory variables. Results. The NHA made 1444 evaluations. For CM, only the Social Security Administration had higher \%RANC than private centers ( $\beta=7.7 \% ; 95 \% \mathrm{Cl} 3.5$ to 11.9$)$. The HCSI of the coastal region $(\beta=-5.2,95 \% \mathrm{Cl}-9.4$ to -1.0$)$, andean region $(\beta=-12.5 ; 95 \% \mathrm{Cl}-16.7$ to -8.3$)$ and jungle region $(\beta=-12.6,95 \% \mathrm{Cl} \%-17.7$ to -7.6) had lower \%RANC than those located in Lima Metropolitan area. \%RANC was higher in 2015 than 2013 ( $\beta=10.8 ; 95 \%$ $\mathrm{Cl} 6.4$ to 15.3). Conclusions. The \%RANC differs by health subsector, region and year of supervision. For CM, the HCSI in the Social Security Administration and in the Lima Metropolitan area had better scores, and scores improved over time. The implementation of actions aimed at improving \%RANC in order to foster the full exercise of health rights in the PHS is suggested.
\end{abstract}

Key words: Risk management; Health centers; Right to health; Health services; Peru (Source: MeSH NLM)

\footnotetext{
Intendencia de Investigación y Desarrollo, Superintendencia Nacional de Salud (SUSALUD). Lima, Perú

Centro de investigación en Salud Pública, Instituto de Investigación, Facultad de Medicina, Universidad de San Martín de Porres. Lima, Perú. Intendencia de Supervisión de Instituciones Prestadoras de Servicios de Salud, Superintendencia Nacional de Salud (SUSALUD). Lima, Perú.

Escuela de Medicina, Universidad Peruana de Ciencias Aplicadas. Lima, Perú.

Superintendencia Adjunta de Supervisión, Superintendencia Nacional de Salud (SUSALUD). Lima, Perú.

Superintendencia Nacional de Salud (SUSALUD). Lima, Perú.

Escuela de Posgrado, Universidad Peruana de Ciencias Aplicadas. Lima, Perú.

Médico cirujano; ${ }^{\mathrm{b}}$ maestro en Ciencias en Investigación Epidemiológica; ${ }^{\mathrm{c}}$ ingeniero estadístico e informático; ${ }^{\mathrm{d}}$ maestro en Ciencias en Epidemiología Clínica; ${ }^{\mathrm{e}}$ especialista en Administración de Salud y Auditoría Médica; ${ }^{\mathrm{f}}$ licenciado en Computación; ${ }^{\mathrm{g}}$ magíster en Ingeniería de Sistemas; ${ }^{\mathrm{h}}$ magíster en Salud Pública; ${ }^{\mathrm{i}}$ maestría en Administración de Negocios.

Recibido: 14/05/2016 Aprobado: 24/08/2016
}

Citar como: Benites-Zapata VA, Saravia-Chong HA, Mezones-Holguin E, Aquije-Díaz A, Villegas-Ortega J, Rossel-de-Almeida G, et al. Modelo de supervisión basado en el riesgo para instituciones prestadoras de servicios de salud como herramienta para la protección de los derechos en salud en Perú. 2016;33(3):401-10. doi:10.17843/rpmesp.2016.333.2373 


\section{INTRODUCCIÓN}

La supervisión del trabajo realizado en una institución prestadora de servicios de salud (IPRESS) contribuye positivamente en el correcto funcionamiento de la misma y busca garantizar la calidad de la asistencia brindada ${ }^{(1)}$. Una atención en salud de calidad conlleva al logro del ejercicio pleno de los derechos en salud (DES) de los ciudadanos ${ }^{(2,3)}$. La finalidad de supervisar radica en identificar los problemas en los procesos de la atención en salud que podría repercutir de manera negativa en los clientes internos y externos de la organización (4). La información generada en la supervisión permite tomar decisiones adecuadas e implementar estrategias de mejora en los procesos de la organización de salud de modo que se puedan prevenir o modificar situaciones que pongan en riesgo la calidad de la atención de salud y vulneren los DES ${ }^{(5,6)}$. En el Perú, la Superintendencia Nacional de Salud (SUSALUD), creada en el año 2013, tiene por mandato legal la misión de proteger los DES en todo el ámbito nacional; en ese marco, una de sus principales funciones es el supervisar a las IPRESS públicas, privadas y mixtas del sistema de salud peruano (SSP) (7). En consecuencia, la labor de SUSALUD contribuye a la mejora del SSP.

Existen diversas normas legales que regulan los procesos de las actividades asistenciales que deben ser cumplidas por las IPRESS. La correcta y oportuna identificación de las contravenciones a estas normas y la posibilidad de generar un determinado riesgo derivado del no cumplimiento adecuado de los procesos asistenciales, convierten a la supervisión en una intervención de alto valor para la prevención de ocurrencias negativas y en una herramienta que favorece el desarrollo de una gestión exitosa. Debido al gran número de IPRESS, - al año 2015 superaban las 20 mil inscritas ${ }^{(8)}$ y al limitado tiempo para efectuar una supervisión presencial, resulta necesario contar con una metodología de supervisión que permita hacer más eficiente dicha tarea. En ese contexto, SUSALUD ha desarrollado instrumentos para supervisar los procesos específicos y el riesgo operacional inherente a ellos, bajo la lógica de que el incumplimiento normativo por parte de las IPRESS incrementa la probabilidad de ocurrencia de riesgos que amenacen la calidad de las prestaciones brindadas. Estas estrategias son novedosas y se vienen implementando progresivamente en el SSP, aunque aún no han sido difundidas en el ámbito académico. Por ello, brindar un panorama general de esta iniciativa y el exponer algunos de sus principales resultados es importante en el campo de la investigación de los servicios de salud en Perú $(9,10)$

El objetivo de este estudio fue describir el modelo de supervisión de las IPRESS de SUSALUD, y evaluar los factores asociados al porcentaje de cumplimiento normativo ajustado a riesgo (\%CNAR) en las IPRESS supervisadas por SUSALUD en el SSP.

\section{MATERIALES Y MÉTODOS}

\section{DISEÑO DE ESTUDIO}

Se llevó a cabo un estudio de caso de la experiencia de SUSALUD en el desarrollo de instrumentos y metodología de supervisión utilizada para obtener el \%CNAR en la supervisión de IPRESS durante los años 2013 a 2015. También, se realizó un estudio de diseño transversal para valorar los factores asociados al $\%$ CNAR en aquellas IPRESS que fueron supervisadas por parte de SUSALUD durante el citado periodo.

\section{ELABORACIÓN DE LOS INSTRUMENTOS DE SUPERVISIÓN DE SUSALUD}

Durante el año 2013 se creó el primer instrumento de supervisión basado en el \%CNAR a cargo de la Intendencia de Supervisión de Instituciones Prestadoras de Salud de SUSALUD (ISIPRESS). Para dicha elaboración, en una primera etapa, se tomó en cuenta las apreciaciones del personal que trabajaba como supervisores, en virtud a su conocimiento empírico de los problemas en el cumplimiento normativo de las IPRESS que supervisaban. Estos profesionales de la salud contaban con estudios de especialización en salud pública o gestión de la calidad o gestión de servicios de salud o de políticas públicas en salud, aseguramiento universal en salud (AUS), así como conocimientos en legislación, desarrollo, implementación, supervisión - monitoreo, auditoria o asistencia técnica. Se construyeron los verificadores en base al marco normativo encontrado y al mapa de procesos elaborado por el equipo técnico responsable de la elaboración del instrumento, este se basó en el marco normativo constituido por 57 dispositivos legales vigentes del Ministerio de Salud (MINSA), la Ley Marco de AUS (Ley 29344, la Ley de los Derechos de las Personas Usuarias de los Servicios de Salud (Ley 29414), la Ley General de Salud (Ley 26842), entre otras normas conexas.

Posteriormente, se agruparon los verificadores en componentes o macroprocesos, con el propósito no solo de conocer el riesgo individual que representa el incumplimiento de un verificador, sino que la suma de incumplimientos (verificadores observados) dentro de un componente represente también el riesgo del componente al cual pertenecen. Para priorizar los componentes dentro del instrumento se empleó la técnica del diagrama de Pareto (11) basado en datos de supervisiones previas (Figura 1). Luego, el instrumento fue enviado a expertos en la especialidad para su validación (sociedades científicas y consultores externos). Los validadores externos debían ser profesionales de la salud con 


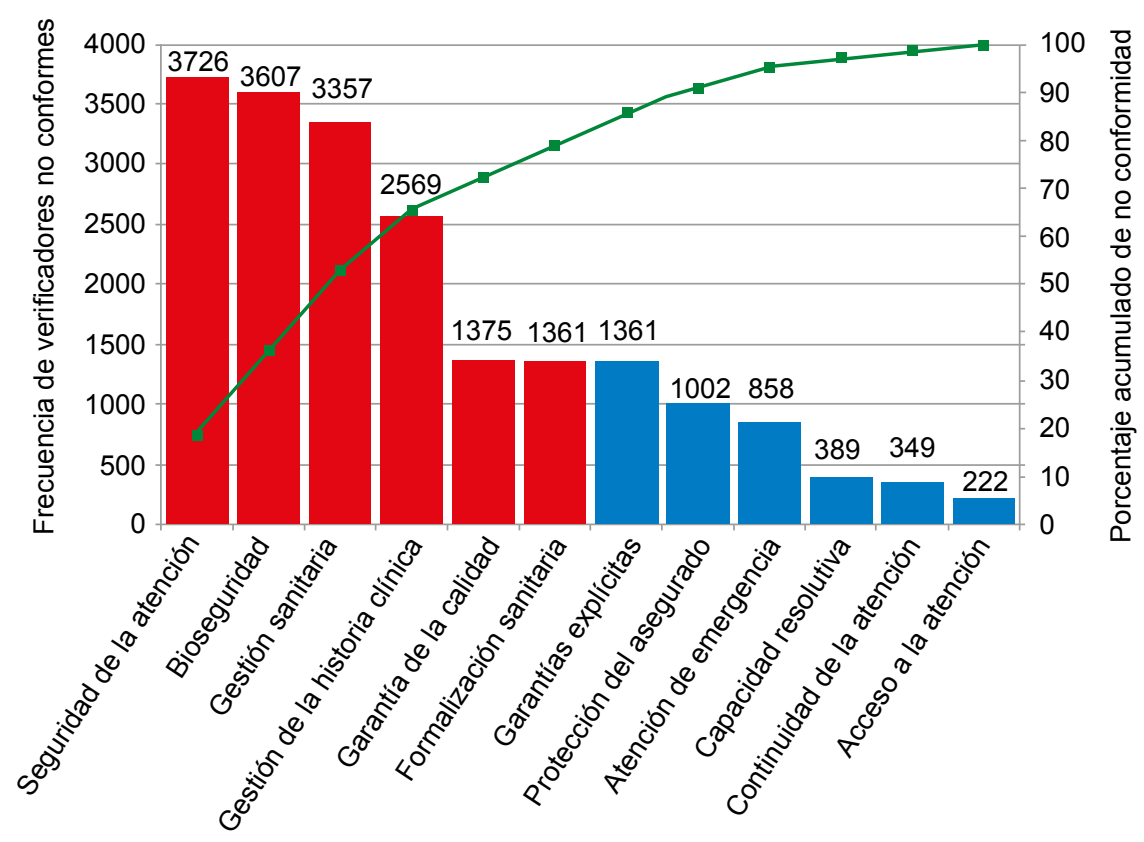

Figura 1. Diagrama de Pareto para la priorización de los componentes de los instrumentos de supervisión de las instituciones prestadoras de servicios de salud (IPRESS) Supeintendencia Nacional de Salud (SUSALUD)

experiencia en el tema, especialistas registrados en el Colegio Médico del Perú y miembros titulares de las sociedades científicas o profesionales con experticia como directores o gerentes de IPRESS.

Para la validación en campo, previamente se llevó a cabo la socialización del instrumento con los supervisores a fin de disminuir la variabilidad en cada medición (repetibilidad) y la variabilidad que aportan entre supervisores (reproductibilidad) ${ }^{(12,13)}$. Se realizaron reuniones de estandarización de procesos con los supervisores que serían los encargados de aplicar el instrumento en la validación de campo. Las reuniones tuvieron una duración de tres días y se realizaron en un lugar apartado a fin de evitar elementos distractores que pudieran interferir en el proceso de estandarización. Después de ello, durante el año 2013 se envió a los supervisores a tres IPRESS seleccionadas por conveniencia: a las dependencias administrativas del MINSA, a la Seguridad Social (EsSalud) y al sector privado. Se compararon los puntajes obtenidos y se utilizaron pruebas estadísticas como el análisis de varianza (ANOVA) de medidas repetidas para verificar las diferencias intraobservador y el coeficiente kappa para valorar la concordancia entre observadores. Adicionalmente, se estimaron los tiempos de aplicación promedio del instrumento durante el trabajo de campo en las IPRESS. En la actualidad, este proceso de estandarización a los supervisores de las IPRESS se lleva a cabo dos veces al año.

El primer instrumento de supervisión creado por ISIPRESS fue de carácter integral, posteriormente se elaboraron herramientas específicas, las cuales consideraron los aspectos identificados mediante las supervisiones desarrolladas con el instrumento de supervisión integral. Para la priorización en la creación de los instrumentos de supervisión selectiva (ISS) se usaron las unidades prestadoras de servicios de salud (UPSS) con el mayor número de incumplimientos en las supervisiones integrales. De ese modo, ISIPRESS desarrolló ISS dirigidos a unidad de cuidados intensivos (UCI), emergencia, farmacia, patología clínica, unidad de hemodiálisis, y primer nivel de atención. Todos los instrumentos están publicados y son de acceso libre en el portal institucional de SUSALUD (http://portales. susalud.gob.pe/web/cdi/estandares-supervision).

Para el año 2014, y basado en la experiencia previa, se publicó la Guía metodológica para la elaboración de instrumentos de supervisión, aprobada mediante Resolución de Superintendencia 043-2014-SUPERINTENDENCIA NACIONAL DE SALUD/S ${ }^{(14)}$. La guía contempla 14 etapas, las cuales son resumidas en la Figura 2.

\section{METODOLOGÍA DEL CÁlCULO DEL PORCENTAJE DE CUMPLIMIENTO NORMATIVO AJUSTADO A RIESGO DE LAS INSTITUCIONES PRESTADORAS DE SERVICIOS DE SALUD}

Luego de la validación del instrumento de supervisión se procedió a ponderar los verificadores para cada componente asignándole un nivel de exposición al riesgo (NER). La ponderación de los verificadores y el cálculo del NER se basaron en una adaptación de la 
norma internacional para la gestión de riesgo ISO:31000$2009^{(15,16)}$. El equipo técnico responsable de elaborar el instrumento de supervisión realizó la ponderación de los verificadores, en base al incumplimiento de los verificadores como riesgo y con la identificación de amenazas por cada riesgo. Para lo cual, se calificó el nivel de impacto y la probabilidad de que se materialice la amenaza ante la presencia de un riesgo.

Para evaluar el impacto se propusieron cinco niveles sobre los cuales el riesgo podría influir: impacto legal, impacto sobre la salud del asegurado, impacto sobre la economía del asegurado, impacto operacional, e impacto sobre la imagen institucional. El equipo técnico calificó dichas amenazas mediante una escala de Likert con puntuaciones que oscilan entre 1 y 5 , donde el valor inferior correspondió a "muy bajo impacto" y el valor superior a "muy alto impacto". Para la calificación del nivel de impacto y la probabilidad de que se materialice la amenaza ante la presencia de un riesgo, se usaron inicialmente diez personas del equipo responsable en la asignación de puntajes de riesgo para cada incumplimiento de los verificadores. Luego de realizar las pruebas estadísticas para determinar si existieron diferencias significativas al utilizar las tres ponderaciones diferentes de riesgo, se estimó que con un mínimo de cinco personas se obtenían los mismos resultados. El nivel de impacto de cada verificador se obtuvo del promedio de los valores de los cinco niveles incluidos.

Para el cálculo de la probabilidad de ocurrencia del riesgo se tomó como referencia el histórico de eventos del año previo en las IPRESS. Dicha probabilidad fue tipificada a través de una escala de Likert con cinco niveles, donde el valor inferior definía una probabilidad menor a $25 \%$, y el valor superior correspondía a un $90 \%$. El instrumento permite que, con el producto de la probabilidad de ocurrencia del riesgo y el nivel de impacto de cada verificador, se obtenga el NER (Figura 3).

Para obtener el puntaje global de las IPRESS supervisadas se suman los niveles de exposición al riesgo de todos los verificadores aplicados contenidos en el instrumento de supervisión. El resultado final se denomina porcentaje de cumplimiento normativo ajustado a riesgo (\%CNAR) y se visualiza en el sistema como un semáforo. El color verde corresponde a un bajo riesgo (>80\% de cumplimiento) lo que representaría una baja posibilidad de ocurrencia y un daño menor en la atención y seguridad del paciente. Por su parte, los colores amarillo (60 a $80 \%$ ) y rojo $(<60 \%)$ corresponden a un mediano y alto riesgo, lo cual conllevaría a una

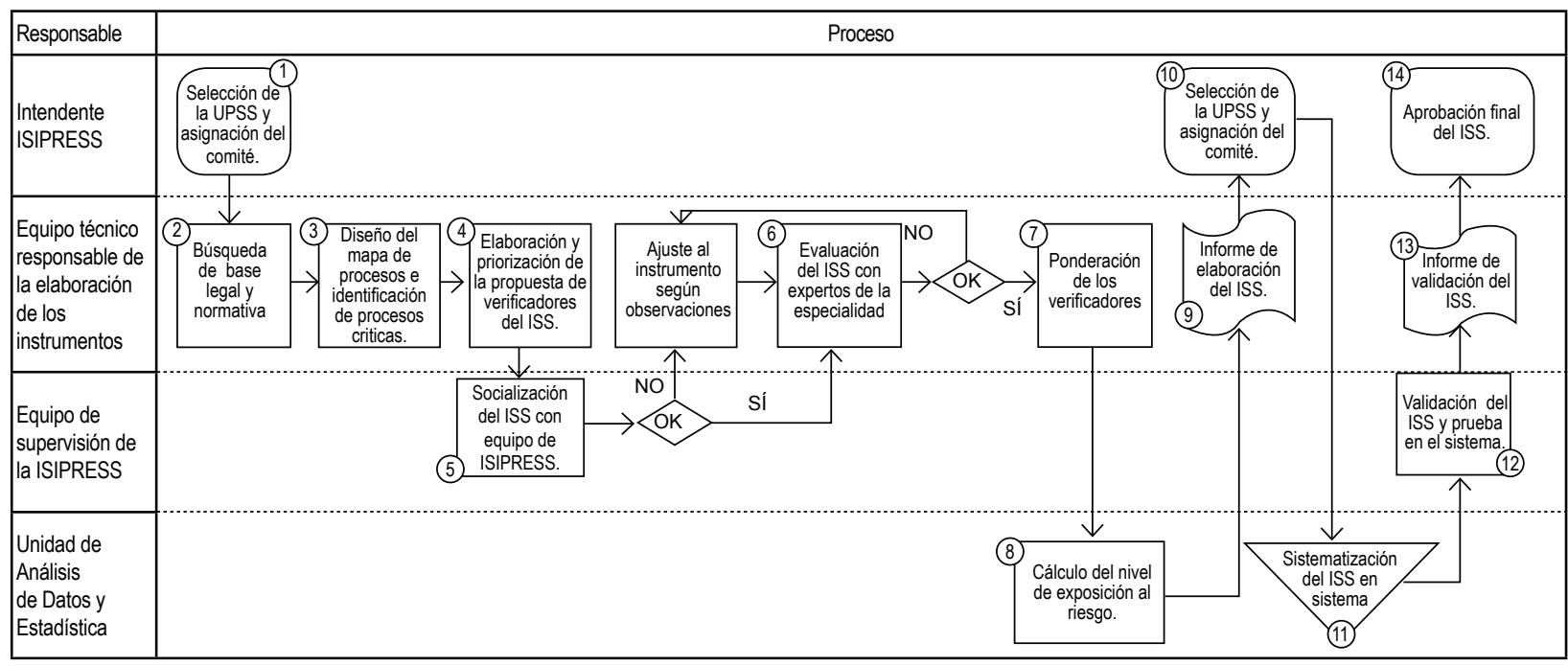

\begin{tabular}{|l|l|l|l|l|l|l|}
\hline Símbolo & & & & \\
\hline Significado & $\begin{array}{l}\text { Indica el inicio y el final } \\
\text { del diagrama de flujo. }\end{array}$ & $\begin{array}{l}\text { Símbolo de proceso, } \\
\text { representa la realización de } \\
\text { una operación o actividad } \\
\text { relativas a un procedimiento. }\end{array}$ & $\begin{array}{l}\text { Representa cualquier tipo } \\
\text { de documento que entra, } \\
\text { se utilice, se genere o } \\
\text { salga del procedimiento. }\end{array}$ & $\begin{array}{l}\text { Indica el depósito } \\
\text { permanente de undica un punto dentro } \\
\text { documento o información } \\
\text { dentro de un archivo. }\end{array}$ & $\begin{array}{l}\text { Inecta los símbolos } \\
\text { del flujo en que son } \\
\text { posibles varios caminos } \\
\text { alternativos. }\end{array}$ \\
$\begin{array}{l}\text { señalando el orden en } \\
\text { que se deben realizar las } \\
\text { distintas operaciones }\end{array}$ \\
\hline
\end{tabular}

SUSALUD: Superintendencia Nacional de Salud, ISIPRESS: Intendencia de Supervisión de Instituciones Prestadoras de Servicios de Salud, ISS: instrumento de supervisión selectiva, UPSS: Unidad Productora de Servicios de salud.

Figura 2. Diagrama de flujo para la elaboración de instrumentos aplicados en Supervisión Selectiva de las Unidades Productoras de servicios de Salud. 
mayor probabilidad de ocurrencia y mayor incremento en la posibilidad de daño mayor en la atención y seguridad del paciente. En resumen, un mayor \% CNAR, se corresponde con un menor riesgo operacional.

Es importante mencionar que la categorización final del riesgo por niveles bajo, medio y alto se decidió con los miembros del equipo después de la revisión de los siguientes documentos técnicos: Resolución Ministerial (R.M.) 669/2005-MINSA, Norma Técnica de Supervisión Integral (035-MINSA/DGSP-V.01), R.M. 672-2009/MINSA que aprueba la Directiva Administrativa de Supervisión Integral a Direcciones de Salud y Direcciones Regionales de salud (158-MINSA/DGSP-V.01), Guía Técnica para el Supervisor Nacional en Salud (R.M. 876-2009/MINSA), Instrumento de Supervisión Integral del MINSA (anexo a la RM 672-2009/MINSA), Resolución Directoral 002-2011GRC/GRS/DIRESA/DG, y el Plan Anual de Supervisión Integral 2011 de la DIRESA Callao.

\section{POBLACIÓN DE ESTUDIO}

La unidad de análisis del estudio fue la IPRESS supervisada. Se incluyeron IPRESS de cualquiera de los diferentes subsectores del SSP, las cuales hayan sido evaluadas mínimamente por dos supervisores. Se excluyeron aquellas IPRESS que tenían más de una evaluación durante el periodo analizado y aquellas que no contaban con los valores correspondientes a todas las variables de interés del estudio. El tamaño de muestra de las IPRESS para supervisión en cada año de estudio se basó en el valor promedio del \%CNAR y su desviación estándar, con un nivel confianza al $95 \%(Z=1,96)$ y un error de $5 \%$. La selección de las IPRESS se realizó mediante muestreo probabilístico estratificado con afijación proporcional. Se consideraron como estratos la región y el nivel de complejidad de la IPRESS.

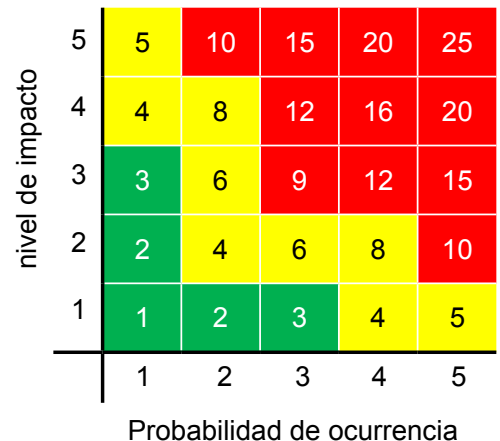

Para el cálculo del nivel de exposición a riesgo para cualquier incumplimiento normativo de un macroproceso se cruzan los valores asignados del nivel de impacto versus la probabilidad de ocurrencia. La zona en verde corresponde a un menor nivel de exposición a riesgo, la zona amarilla corresponde a un intermedio nivel de exposición a riesgo y la zona roja corresponde a un alto nivel de exposición a riesgo.

Figura 3. Matriz para el cálculo del nivel de exposición a riesgo para incumplimiento normativo

\section{VARIABLES PARA EL ESTUDIO DE FACTORES ASOCIADOS AL PORCENTAJE DE CUMPLIMIENTO NORMATIVO AJUSTADO A RIESGO}

La variable de respuesta fue el \%CNAR. Los tipos de supervisión fueron la integral y la supervisión selectiva (basada en UPSS). La supervisión integral se definió como aquellas supervisiones que involucran todos los servicios o UPSS de la IPRESS. Las UPSS son las unidades dentro de las IPRESS organizadas en sus procesos para prestar servicios de salud, dichas unidades cuentan con infraestructura, equipamiento, medicamentos, procedimientos clínicos, así como de personal de salud y recursos tecnológicos. En ese sentido, cuando en el artículo se hace referencia a la supervisión de emergencias, $\mathrm{UCl}$, patología clínica o farmacia; se refiere específicamente a solo la UPSS organizada para dar ese servicio.

Las variables de exposición fueron el subsector de salud categorizado en MINSA y gobiernos regionales (MINSAGR), EsSalud, Sanidades de las Fuerzas Armadas y Policiales (SSFFAAPP) y el subsector privado; el nivel de complejidad de la IPRESS de acuerdo al registro nacional de IPRESS de SUSALUD; la región donde se encuentra ubicada la IPRESS conformada por Lima Metropolitana, la costa, la sierra y la selva; y finalmente el año en que se llevó a cabo la supervisión.

Es importante mencionar que el tipo de supervisión fue considerado como un estrato para el desarrollo de un modelo estadístico específico.

\section{ANÁLISIS DE DATOS}

Se presenta las características generales de las IPRESS por medio de frecuencias y porcentajes. Las comparaciones entre las categorías del \%CNAR se llevaron a cabo utilizando la prueba de ji cuadrado o la prueba exacta de Fisher, de acuerdo a la evaluación de los valores esperados. Si bien es posible utilizar la variable \%CNAR categorizada en riesgo bajo, moderado y alto para llevar a cabo un análisis de regresión logística ordinal o multinomial, por cuestiones de consistencia estadística debido a que algunos estratos de la variable de respuesta tenían denominadores pequeños y ello desestabilizaba el modelo, se decidió analizar el $\%$ CNAR como una variable numérica.

Para valorar los factores asociados al \%CNAR se desarrollaron modelos lineales generalizados (MLG) de múltiples variables de la familia gaussiana con función de enlace de identidad, con lo que estimamos el coeficiente Beta $(\beta)$ con su respectivo intervalo de confianza (IC) al 95\% como medida de asociación. En los MLG fueron verificados los supuestos de normalidad, homocedasticidad de la 
varianza, homogeneidad del modelo e independencia; ello con la finalidad de que la valoración de la asociación entre el desenlace principal y las variables de exposición sea apropiada ${ }^{(17-19)}$. Tras ello, se consideraron solo los siguientes tipos de supervisión: integral, emergencias y UCI Dado que lo que se buscaba era establecer factores asociados y cuantificar las diferencias entre los estratos evaluados se decidió ingresar al MLG las variables sector de salud, región de procedencia de la IPRESS, nivel de complejidad y año de realización de la supervisión como variables de exposición a priori. Debido a que todas las variables de exposición en el MLG son categóricas, coeficiente $\beta$ se interpreta como la diferencia en el \%CNAR respecto del estrato de referencia seleccionado ajustada por las otras covariables del modelo. Los análisis se efectuaron en el paquete estadístico STATA versión 12.1 (StataCorp, TX, USA).

\section{RESULTADOS}

Se incluyeron un total de 285 supervisiones en el año 2013, 425 supervisiones en el año 2014 y 734 supervisiones en el año 2015. La mayoría de supervisiones realizadas fueron a las IPRESS del MINSA-GR. En cada uno de los años estudiados, la mayor proporción de las IPRESS supervisadas fueron de Lima Metropolitana. En todo el periodo, el tipo de supervisión más frecuente fue la supervisión integral. Las características de las IPRESS según el año de supervisión se presentan en la Tabla 1.

La Tabla 2 muestra las características de las IPRESS según el nivel de riesgo derivado del \%CNAR. Se observa que la frecuencia de alto riesgo fue de 57,3\% (404/705) en las IPRESS del MINSA-GR; 38,3\% (97/253) para las IPRESS de EsSalud; 46,4\% (33/71) y 36,6\% (152/415) para las IPRESS del subsector privado $(p<0,01)$. En cuanto a la ubicación geográfica de la IPRESS, se observó que el 38,1\% (303/796) de las IPRESS de Lima Metropolitana se encontraban en alto riesgo, en contraste con el $70 \%$ $(71 / 101)$ para las IPRESS de la selva $(p<0,01)$. También, se encontró que el 67\% (237/355) de las IPRESS en el primer nivel de atención fueron catalogadas como de alto riesgo, en cambio de las IPRESS supervisadas en el tercer nivel de atención solo el $25,5 \%$ poseyeron esa categoría $(p<0,01)$. La supervisión integral encontró al 60,7\% (314/507) de las IPRESS con alto riesgo. En oposición, la supervisión de centros de hemodiálisis (9/72) y banco de sangre (8/62) fueron las UPSS con el menor porcentaje de alto riesgo, el cual estuvo alrededor del $12 \%(p<0,01)$. Se distinguió una disminución de las IPRESS calificadas como de alto riesgo durante el año 2015 (249/734) con respecto al año 2013 (191/285) y del mismo modo se puede apreciar un aumento de las IPRESS calificadas como de bajo riesgo para el año 2015 (202/734) comparado con el año 2013 (20/285).
La Tabla 3 presenta los MLG de múltiples variables para cada uno de los tres tipos de supervisión que cumplieron los supuestos matemáticos correspondientes: integral, de emergencia y de $\mathrm{UCl}$.

En el MLG de múltiples variables para el tipo de supervisión integral con los centros privados como estrato de referencia se repara que EsSalud tiene el promedio mayor de \%CNAR ( $\beta=7,7 \%$; IC95\%: 3,5 a 11,9), en cambio no se advirtieron diferencias con el MINSA-GR y las SSFFAAPP. Del mismo modo, utilizando a Lima Metropolitana como estrato de referencia se encontró que hay en promedio un menor \%CNAR para las IPRESS en las regiones de la costa ( $\beta=-5,2$; IC $95 \%$ : $-9,4$ a $-1,0)$,

Tabla 1. Características generales de las Instituciones Prestadoras de Servicios de Salud (IPRESS) según Año de supervisión por la Superintendencia Nacional de Salud (SUSALUD) por año de estudio

\begin{tabular}{|c|c|c|c|}
\hline \multirow[t]{2}{*}{ Variables } & $\begin{array}{c}2013 \\
n=285\end{array}$ & $\begin{array}{c}2014 \\
n=425\end{array}$ & $\begin{array}{c}2015 \\
n=734\end{array}$ \\
\hline & $\mathrm{n}(\%)$ & $\mathrm{n}(\%)$ & $\mathrm{n}(\%)$ \\
\hline \multicolumn{4}{|l|}{ Subsector Supervisado } \\
\hline MINSA-GR & $189(66,3)$ & $194(45,7)$ & $322(43,9)$ \\
\hline ESSALUD & $27(9,5)$ & $69(16,2)$ & $157(21,4)$ \\
\hline SSFFAAPP & $11(3,9)$ & $23(5,4)$ & $37(5,0)$ \\
\hline Privados & $58(20,3)$ & $139(32,7)$ & $218(29,7)$ \\
\hline \multicolumn{4}{|l|}{ Región geográfica } \\
\hline Lima Metropolitana & $166(58,2)$ & $285(67,0)$ & $345(47,0)$ \\
\hline Costa & $71(24,9)$ & $96(22,6)$ & $188(25,6)$ \\
\hline Sierra & $23(8,1)$ & $33(7,8)$ & $136(18,5)$ \\
\hline Selva & $25(8,8)$ & $11(2,6)$ & $65(8,9)$ \\
\hline \multicolumn{4}{|l|}{ Categoría RENIPRESS } \\
\hline Primer nivel & $155(54,4)$ & $178(41,9)$ & $21(2,9)$ \\
\hline Segundo nivel & $111(39)$ & $174(40,9)$ & $591(80,6)$ \\
\hline Tercer nivel & $7(2,5)$ & $19(4,5)$ & $72(9,8)$ \\
\hline SMA & $12(4,2)$ & $10(2,4)$ & $18(2,5)$ \\
\hline Sin categoría & $0(0)$ & $44(10,3)$ & $31(4,2)$ \\
\hline \multicolumn{4}{|l|}{ Tipo Supervisión } \\
\hline Integral & $176(62,0)$ & $149(35,1)$ & $192(26,2)$ \\
\hline Primer nivel & $43(15,1)$ & $131(30,8)$ & $11(1,5)$ \\
\hline Emergencia & $53(18,7)$ & $5(1,2)$ & $110(15,0)$ \\
\hline Cuidados intensivos & $0(0,0)$ & $85(20,0)$ & $150(20,4)$ \\
\hline Patología Clínica & $0(0,0)$ & $13(3,1)$ & $83(11,3)$ \\
\hline Farmacia & $0(0,0)$ & $3(0,7)$ & $95(12,9)$ \\
\hline Hemodiálisis & $12(4,2)$ & $30(7,1)$ & $30(4,1)$ \\
\hline $\begin{array}{l}\text { Diagnóstico por } \\
\text { imágenes }\end{array}$ & $0(0,0)$ & $9(2,1)$ & $1(0,1)$ \\
\hline Banco de sangre & $0(0,0)$ & $0(0,0)$ & $62(8,5)$ \\
\hline
\end{tabular}

MINSA: Ministerio de Salud, GR: Gobiernos Regionales, ESSALUD: Seguridad Social en Salud, SSFFAAPP: Sanidades de las Fuerzas Armadas y Policiales, RENIPRESS: Registro Nacional de Instituciones Prestadoras de Servicios de Salud, SMA: servicios médicos de apoyo. 
sierra $(\beta=-12,5$; IC95\%: $-16,7$ a $-8,3)$ y selva $(\beta=-12,6$; IC95\%: $-17,7$ a $-7,6)$; respectivamente. También, se evidenció que, en el primer nivel de complejidad como base, no hubo diferencias respecto del promedio del $\%$ CNAR con los diferentes niveles de complejidad, ni con los servicios médicos de apoyo (SMA); no obstante, sí hubo, en promedio, un menor \%CNAR en las IPRESS que no tenían categorización según el RENIPRESS ( $\beta=$ 12,5; IC95\%: -20,6 a -4,3). A su vez, con el año 2013 como categoría de referencia, se encontró en promedio, un mayor \%CNAR durante el año 2015, ( $\beta=10,8$; IC95\%: 6,4 a 15,3) sin evidencia estadística con el año 2014.

Para el tipo de supervisión en emergencia se evidenció que tanto MINSA-GR ( $\beta=-20,1$; IC95\%: $-26,7$ a $-13,6)$, EsSalud ( $\beta=-10,1$; IC95\%: $-17,7$ a $-2,5)$ y las SSFFAAPP ( $\beta=-29,7$; IC95\%: $-43,6$ a -15,8) tienen en promedio, menor $\%$ CNAR que los centros privados. Asimismo, se encontró que hay en promedio un menor \%CNAR para las IPRESS en las regiones de la costa ( $\beta=-14,3$; IC95\%: $-20,1$ a -8,4) y selva $(\beta=-14,4$; IC95\%: $-26,2$ a $-2,6)$ con respecto a Lima Metropolitana. También, se constató que no hubo diferencias de acuerdo al nivel de complejidad. Además, se observó en promedio, un mayor \%CNAR en el año 2015 ( $\beta=11,6$; IC 95\%: 5,8 a 17,3) en relación al año 2013.

Para la supervisión de UCI se encontró que tanto el MINSAGR ( $\beta=-12,3$; IC95\%: -16,2 a -8,4), EsSalud $(\beta=-9,9$; IC95\%: $-14,8$ a $-5,0)$ y las SSFFAAPP $(\beta=-6,6$; IC95\%: -12,2 a $-1,0)$ tienen en promedio, menor \%CNAR que los centros privados. Del mismo modo, se apreció que las IPRESS en las regiones de la costa ( $\beta=-6,5$; IC95\%: -11,4 a -1,6), sierra $(\beta=-7,7$; IC95\%: $-13,4$ a $-2,1)$ y selva $(\beta=-15,8$; IC95\%: $-22,6$ a $-8,9)$ tienen en promedio un menor \%CNAR que las ubicadas en Lima Metropolitana. Asimismo, se observó que el tercer nivel de complejidad presentó en promedio, mayor

Tabla 2. Características de las instituciones prestadoras de servicios de salud supervisadas (IPRESS) por la Superintendencia Nacional de Salud (SUSALUD) de acuerdo a su nivel de riesgo (2013-2015)

\begin{tabular}{|c|c|c|c|c|}
\hline \multirow[t]{2}{*}{ Variables } & $\begin{array}{c}\text { Bajo Riesgo } \\
n=263\end{array}$ & $\begin{array}{c}\text { Mediano Riesgo } \\
\mathrm{n}=495\end{array}$ & $\begin{array}{c}\text { Alto Riesgo } \\
n=686\end{array}$ & \multirow{2}{*}{$\begin{array}{c}\text { Valor } \\
p\end{array}$} \\
\hline & $\mathrm{n}(\%)$ & $\mathrm{n}(\%)$ & $n(\%)$ & \\
\hline Subsector supervisado & & & & $<0,01$ \\
\hline MINSA-GR & $66(9,4)$ & $235(33,3)$ & $404(57,3)$ & \\
\hline ESSALUD & $56(22,1)$ & $100(39,5)$ & $97(38,4)$ & \\
\hline SSFFAAPP & $10(14,1)$ & $28(39,4)$ & $33(46,5)$ & \\
\hline Privados & $131(31,6)$ & $132(31,8)$ & $152(36,6)$ & \\
\hline Región geográfica & & & & $<0,01$ \\
\hline Lima Metropolitana & $203(25,5)$ & $290(36,4)$ & $303(38,1)$ & \\
\hline Costa & $41(11,5)$ & $117(33)$ & $197(55,5)$ & \\
\hline Sierra & $15(7,8)$ & $62(32,3)$ & $115(59,9)$ & \\
\hline Selva & $4(4,0)$ & $26(25,7)$ & $71(70,3)$ & \\
\hline Categoría (RENIPRESS) & & & & $<0,01$ \\
\hline Primer nivel & $10(2,8)$ & $108(30,2)$ & $237(67)$ & \\
\hline Segundo nivel & $186(21,2)$ & $314(35,9)$ & $376(42,9)$ & \\
\hline Tercer nivel & $36(36,7)$ & $37(37,8)$ & $25(25,5)$ & \\
\hline SMA & $14(35,0)$ & $10(25,0)$ & $16(40,0)$ & \\
\hline Sin categoría & $17(22,6)$ & $26(34,7)$ & $32(42,7)$ & \\
\hline Tipo de supervisión & & & & $<0,01$ \\
\hline Integral & $50(9,7)$ & $153(29,6)$ & $314(60,7)$ & \\
\hline Primer nivel & $1(0,5)$ & $45(24,3)$ & $139(75,2)$ & \\
\hline Emergencia & $25(14,9)$ & $51(30,4)$ & $92(54,7)$ & \\
\hline Cuidados intensivos & $66(28,1)$ & $116(49,4)$ & $53(25,5)$ & \\
\hline Patología clínica & $26(27,1)$ & $37(38,5)$ & $33(34,4)$ & \\
\hline Farmacia & $31(31,6)$ & $34(34,7)$ & $33(33,7)$ & \\
\hline Hemodiálisis & $35(48,6)$ & $28(38,9)$ & $9(12,5)$ & \\
\hline Diagnóstico por imágenes & $0(0,0)$ & $5(50,0)$ & $5(50,0)$ & \\
\hline Banco de sangre & $29(46,8)$ & $25(40,3)$ & $8(12,9)$ & \\
\hline Año de supervisión & & & & $<0,01$ \\
\hline 2013 & $20(7,0)$ & $74(26,0)$ & $191(67,0)$ & \\
\hline 2014 & $41(9,7)$ & $138(32,5)$ & $246(57,8)$ & \\
\hline 2015 & $202(27,5)$ & $283(38,6)$ & $249(33,9)$ & \\
\hline
\end{tabular}

MINSA: Ministerio de Salud, GR: gobiernos regionales, ESSALUD: Seguridad Social en Salud, SSFFAAPP: Sanidades de las Fuerzas Armadas y Policiales, RENIPRESS: Registro Nacional de Instituciones Prestadoras de Servicios de Salud, SMA: servicios médicos de apoyo 
Tabla 3. Factores asociados al porcentaje de cumplimiento normativo ajustado a riesgo en tres tipos de supervisión en las Instituciones prestadoras de servicios de Salud (IPRESS) supervisadas por la Superintendencia Nacional de Salud (SUSALUD).

(Modelos lineales generalizados de múltiples variables de familia gaussiana con función de enlace de identidad)

\begin{tabular}{|c|c|c|c|c|c|c|}
\hline \multirow[t]{2}{*}{ Variables } & \multicolumn{2}{|c|}{ Supervisión Integral } & \multicolumn{2}{|c|}{ Supervisión de Emergencia } & \multicolumn{2}{|c|}{$\begin{array}{l}\text { Supervisión de la Unidad de } \\
\text { Cuidados Intensivos }\end{array}$} \\
\hline & $\beta^{*}($ IC 95\%) & Valor $p$ & $\beta^{*}(\mathrm{IC} 95 \%)$ & Valor $p$ & $\beta^{*}($ IC 95\%) & Valor $p$ \\
\hline \multicolumn{7}{|l|}{ Subsector supervisado } \\
\hline Privados & Referencia & & Referencia & & Referencia & \\
\hline MINSA-GR & $-1,55(-5,2$ a 2,1$)$ & 0,41 & $-20,1(-26,7$ a $-13,6)$ & $<0,01$ & $-12,3(-16,2$ a $-8,4)$ & $<0,01$ \\
\hline ESSALUD & $7,7(3,5$ a 11,9$)$ & $<0,01$ & $-10,1(-17,7$ a $-2,5)$ & 0,01 & $-9,9(-14,8$ a $-5,0)$ & $<0,01$ \\
\hline SSFFAAPP & $-3,7(-11,0$ a 3,6$)$ & 0,32 & $-29,7(-43,6$ a $-15,8)$ & $<0,01$ & $-6,6(-12,2$ a $-1,0)$ & 0,02 \\
\hline \multicolumn{7}{|l|}{ Región geográfica } \\
\hline Lima Metropolitana & Referencia & & Referencia & & Referencia & \\
\hline Costa & $-5,2(-9,4$ a $-1,0)$ & 0,02 & $-14,3(-20,1$ a $-8,4)$ & $<0,01$ & $-6,5(-11,4$ a $-1,6)$ & $<0,01$ \\
\hline Sierra & $-12,5(-16,7$ a $-8,3)$ & $<0,01$ & $-6,8(-15,5$ a 1,9$)$ & 0,12 & $-7,7(-13,4$ a $-2,1)$ & $<0,01$ \\
\hline Selva & $-12,6(-17,7$ a $-7,6)$ & $<0,01$ & $-14,4(-26,2$ a $-2,6)$ & 0,02 & $-15,8(-22,6$ a $-8,9)$ & $<0,01$ \\
\hline \multicolumn{7}{|l|}{ Categoría RENIPRESS } \\
\hline Primer nivel & Referencia & & NA & & NA & \\
\hline Segundo nivel & $-2,3(-6,3$ a 1,7$)$ & 0,26 & Referencia & & Referencia & \\
\hline Tercer nivel & $5,6(-8,3$ a 19,6$)$ & 0,43 & $-0,9(-9,1$ a 7,2$)$ & 0,82 & $5,6(1,1$ a 10,1$)$ & 0,01 \\
\hline SMA & $-7,9(-18,4$ a 2,5$)$ & 0,14 & NA & & NA & \\
\hline Sin categoría & $-12,5(-20,6$ a $-4,3)$ & $<0,01$ & $-2,9(-19,3$ a 13,56$)$ & 0,73 & $7,2(-8,9$ a 23,4$)$ & 0,37 \\
\hline \multicolumn{7}{|l|}{ Año de supervisión } \\
\hline 2013 & Referencia & & Referencia & & NA & \\
\hline 2014 & $-2,8(-7,3$ a 1,6$)$ & 0,21 & $-7,9(-22,5$ a 6,6$)$ & 0,28 & Referencia & \\
\hline 2015 & $10,8(6,4$ a 15,3$)$ & $<0,01$ & $11,6(5,8$ a 17,3$)$ & $<0,01$ & $7,1(3,7$ a 10,4$)$ & $<0,01$ \\
\hline
\end{tabular}

MINSA: Ministerio de Salud, GR: gobiernos regionales, ESSALUD: Seguridad Social en Salud, SSFFAAPP: Sanidades de las Fuerzas Armadas y Policiales, IC: intervalo de confianza, NA: no aplica, RENIPRESS: Registro Nacional de Instituciones Prestadoras de Servicios de Salud, SMA: servicios médicos de apoyo, $\beta$ : coeficiente beta

* Un coeficiente $\beta$ positivo significa que ese estrato de la variable se encuentra en menor riesgo que el estrato de referencia, mientras que un coeficiente $\beta$ negativo significa que ese estrato de la variable se encuentra en mayor riesgo que el estrato de referencia

$\%$ CNAR ( $\beta=5,6$; IC95\%: 1,1 a 10,1) que el primer nivel de atención. A su vez, con el año 2014 como categoría de referencia, se encontró en promedio, un mayor \%CNAR en las $\mathrm{UCl}$ de las IPRESS supervisadas durante el año 2015 $(\beta=7,1 ;$ IC95\%: 3,7 a 10,4$)$.

\section{DISCUSIÓN}

El presente estudio ha permitido exponer el modelo de supervisión basado en el cumplimiento normativo ajustado a riesgo para IPRESS desarrollado por SUSALUD. Esta iniciativa ha supuesto un cambio substancial en la política de supervisión del componente prestacional en el SSP, con la cual se ha dejado atrás el modelo previo donde solo se verificaba el cumplimiento de las normativas legales vigentes para dicho periodo sin asociación alguna con el nivel de riesgo. Este nuevo modelo introduce la caracterización de riesgo dentro de los macroprocesos que llevan a cabo las IPRESS en la realización de su actividad asistencial. Esto permite, que estas organizaciones puedan efectuar acciones que busquen gestionar dichos riesgos con la finalidad de brindar servicios de calidad, lo cual indudablemente contribuye a la generación de un ambiente que favorece el ejercicio de los DES por la ciudadanía.

Dentro de los principales hallazgos se debe mencionar que cuando se llevó a cabo la supervisión de manera integral, solo las IPRESS de EsSalud tenían en promedio, mayor \%CNAR que los centros privados. En cambio, cuando se llevó a cabo la supervisión de las Emergencias y de las UCI, tanto las IPRESS del MINSA-GR, de EsSalud y las de las SSFFAAPP tuvieron menor \%CNAR en comparación con el sector privado. En un marco donde el sistema de salud es fraccionado y fragmentado, la importancia de reconocer cuáles subsectores están en riesgo es cardinal, más aun en un contexto de transición hacia el aseguramiento universal, donde cerca del $80 \%$ de los usuarios del SSP reciben atención en el MINSA-GR y EsSalud ${ }^{(20)}$. Ante ello, los riesgos identificados requieren un abordaje que busque su mitigación o eliminación, con el propósito de brindar una atención de calidad y, con ello, beneficiar la protección de los DES.

Otro hallazgo importante es que las IPRESS que se encuentran fuera de Lima Metropolitana presentan, 
en promedio, menores \%CNAR. Esta información es valiosa dado que muestra que existen mayores deficiencias a nivel prestacional en las zonas ubicadas fuera de la capital, lo cual sugiere que, a pesar de las políticas de descentralización y desconcentración del sector salud, se requieren mayores acercamientos y estrategias a nivel regional, no solo para cumplir con las disposiciones normativas que se exigen, sino también para crecer en la calidad de los servicios que se bridan. Ese marco requiere la promoción de un trabajo conjunto y multidisciplinario por parte de los GR y sus respectivas direcciones regional de salud, con el MINSA y SUSALUD, entre otras instituciones del Gobierno Central, lo cual debería generar estrategias efectivas, consensuadas y contextualizadas para cada región.

En general, no se evidencian diferencias en el \%CNAR entre los niveles de complejidad del sistema de salud. Esta situación debe tomarse en cuenta para priorizar, preferentemente, la mejora del cumplimiento normativo en los primeros niveles de complejidad asistencial, colocando especial énfasis en mitigar los riesgos encontrados, dado que por su naturaleza preventiva y promocional de la salud son una medida más costoefectiva que la actividad recuperativa que se dan en los niveles con mayor nivel de complejidad ${ }^{(21)}$. No obstante, como se ha expresado previamente estas deben considerar los aspectos geopolíticos de cada IPRESS, como son las condiciones del subsector y su ubicación geográfica.

En relación con el periodo, en todos los tipos de supervisión llevados a cabo se ve una mejoría de los \%CNAR en comparación con la línea de base. Dado que este estudio no ha valorado el \%CNAR postsupervisión no podríamos aseverar que la mejoría en el tiempo del \%CNAR se deba a la intervención, sin embargo, es plausible creer que las constantes acciones de SUSALUD hayan podido influenciar en tales hallazgos ${ }^{(22,23)}$. Ello, como un efecto indirecto al requerir estar preparados para una potencial supervisión.

El presente estudio tiene limitaciones, una de las principales es que el uso basal de la norma ISO: 31000 no hace posible comparar nuestros hallazgos con el de otros países de la región, sin embargo, la mirada basada en riesgo es el camino natural que se emplea en América Latina. Otra limitación es que al tener un diseño transversal en el análisis de los datos solo se pueden identificar factores asociados y no necesariamente ellos reflejan relaciones de causalidad. Dado que se trata de un primer abordaje del tópico, el reconocimiento de marcadores puede ser útil y permitiría sentar bases para el desarrollo de futuras investigaciones donde los diseños longitudinales y de series temporales podrían aclarar esos aspectos. Otra limitación es la exclusión de las IPRESS que tuvieron más de una supervisión, esto se optó debido a la concepción de un modelo estadístico que sirva para valorar los factores asociados como marcadores transversales; si bien el análisis de medidas repetidas hubiese aportado información valiosa, la baja proporción de dichas IPRESS no permite aún ejecutar algunos modelos estables para dicho fin. Se sugieren el desarrollo de futuros estudios donde se incorpore este elemento. Asimismo, otra limitación, fue no contar con un muestreo probabilístico con representatividad nacional y regional, no obstante, lo presentado constituye el statu quo de la supervisión de las IPRESS en Perú y es la serie más grande al respecto donde se incluyen instituciones de la capital y de otras regiones del país.

Más allá de las limitaciones expuestas, este manuscrito constituye una primera aproximación y comunicación académica en la que se describe los mecanismos de supervisión que se desarrollan sobre las IPRESS en Perú, y donde se identifica una diversidad de factores asociados al no cumplimiento de la normatividad ajustada al riesgo. Ello conforma un reto importante para los tomadores de decisiones, quienes deben considerar que la situación precisa un trato multidisciplinario, interinstitucional e intrainstitucional para aminorar los riesgos detectados en los procesos de supervisión. Esta canalización de recursos y esfuerzos hacia las IPRESS debe considerar también, que cada una de ellas posee características del subsector, región y nivel de complejidad a la que pertenece, pero también características individuales como el clima organizacional, los recursos humanos y la infraestructura - que requieren una dosis de singularidad en el manejo. Es recomendable que las acciones tomadas sean sustentadas en la mejor evidencia disponible con el propósito de incrementar su probabilidad de éxito; además, se debe contar con la participación de los distintos actores vinculados a las IPRESS, todo ello con un enfoque de equidad, igualdad y la valoración de aspectos financieros que le den sostenibilidad con miras a preservar el derecho que tienen todos los ciudadanos a una atención en salud con calidad ${ }^{(24,25)}$.

Contribuciones de autoría: VBZ, HSC; EMH, JVO, CAS, FPC, AAD participaron en el diseño del estudio y la concepción del artículo. VBZ, AAD contribuyeron con la adquisición de datos. VBZ, AAD y HSC realizaron la escritura del borrador del artículo. VBZ, GRA realizaron la interpretación de datos. Todos hicieron la revisión crítica del artículo, aprobaron la versión final y se hacen responsables del contenido.

Fuente de financiamiento: El presente artículo fue financiado por la Superintendencia Nacional de Salud (SUSALUD)

Conflictos de interés: al momento de la publicación los autores laboran en la Superintendencia Nacional de Salud (SUSALUD). 


\section{REFERENCIAS BIBLIOGRÁFICAS}

1. Ivers N, Jamtvedt G, Flottorp S, Young JM, Odgaard-Jensen J, French SD, et al. Audit and feedback: effects on professional practice and healthcare outcomes. Cochrane Database Syst Rev. 2012;13(6):CD000259. doi: 10.1002/14651858.CD000259.pub3

2. Backman G, Hunt P, Khosla R, Jaramillo-Strouss C, Fikre BM, Rumble C, et al. Health systems and the right to health: an assessment of 194 countries. Lancet. 2008;372(9655):2047-85. doi: 10.1016/S0140-6736(08)61781-X

3. Hernández M. Reforma sanitaria, equidad y derecho a la salud en Colombia. Cad Saúde Pública. 2002;18(4):991-1001. doi:10.1590/ S0102-311X2002000400007

4. Ferlie EB, Shortell SM. Improving the quality of health care in the United Kingdom and the United States: a framework for change. Milbank Q. 2001;79(2):281-315. doi: 10.1111/1468-0009.00206

5. Malik RE, Cooper RA, Griffith CJ. Use of audit tools to evaluate the efficacy of cleaning systems in hospitals. Am J Infect Control. 2003;31(3):181-7. doi:10.1067/mic.2003.34

6. Price LC, Lowe D, Hosker HS, Anstey K, Pearson MG, Roberts CM. UK National COPD Audit 2003: impact of hospital resources and organisation of care on patient outcome following admission for acute COPD exacerbation. Thorax. 2006;61(10):83742. doi:10.1136/thx.2005.049940

7. Perú, Presidencia de la República. Decreto Legislativo 1158. Decreto Legislativo que dispone de medidas destinadas al fortalecimiento y cambio de denominación de la Superintendencia Nacional de Aseguramiento en Salud. (6 de diciembre de 2013).

8. Superintendencia Nacional de Salud (SUSALUD). Anuario Estadístico [Internet]. Lima: SUSALUD; 2015 [citado 23 de agosto de 2016]. Disponible en: http://portales.susalud. gob.pe/documents/102363/129001/ ANUARIO+2016+final+160725.pdf

9. Bernal-Delgado E, Peiró S, Sotoca R. Prioridades de investigación en servicios sanitarios en el Sistema Nacional de Salud. Una aproximación por consenso de expertos. Gac Sanit. 2006;20(4):287-94.
10. Sonis A. Investigación en servicios de salud [Internet]. Buenos Aires: OPS/ OMS; 1989 [citado 23 de agosto de 2016]. Disponible en: http://iris.paho. org/xmlui/handle/123456789/18739

11. Messac A, Ismail-Yahaya A, Mattson CA. The normalized normal constraint method for generating the Pareto frontier. Struct Multidiscip Optim. 2003;25(2):86-98. doi:10.1007/ s00158-002-0276-1

12. Botero Arbeláez M, Arbeláez Salazar O, Mendoza Vargas JA. Método Anova utilizado para realizar el estudio de repetibilidad y reproducibilidad dentro del control de calidad de un sistema de medición [Internet]. Pereira: Universidad Tecnológica de Pereira; 2007 [citado 23 de agosto de 2016]. Disponible en: http://repositorio.utp. edu.co/dspace/handle/11059/321

13. Deyo RA, Diehr P, Patrick DL. Reproducibility and responsiveness of health status measures statistics and strategies for evaluation. Control Clin Trials. 1991 Aug;12(4 Suppl):142S-158S.

14. Perú, Superintendencia Nacional de Salud (SUSALUD). Resolución de Superintendencia 043-2014Superintendencia Nacional de Salud/S [Internet]. Lima: SUSALUD; 2014 [citado el 13 de mayo de 2016]. Disponible en: http://app10.susalud. gob.pe:8080/wb-consulta-documental/ getFicha.htm ? url Arch=/d/d/ workspace/SpacesStore/40a4d05f0365-4454-93a9-47e7401649db/775

15. Leitch M. ISO 31000:200--The New International Standard on Risk Management. Risk Anal. 2010 Jun;30(6):887-92. doi: 10.1111/j.15396924.2010.01397.x.

16. Purdy G. ISO 31000: 2009-setting a new standard for risk management. Risk Anal. 2010 Jun;30(6):881-6. doi: 10.1111/j.1539-6924.2010.01442.x.

17. Schneider A, Hommel G, Blettner M. Linear Regression Analysis. Dtsch Ä Rztebl Int. 2010;107(44):776-82.

18. McCulloch CE. Generalized linear models. J Am Stat Assoc. 2000;95(452):1320-4.

19. Crawley MJ. Generalized linear models. En: Crawley MJ. The R Book. Chichester, UK: John Wiley \& Sons; 2007. p.511-26.
20. Castro J. Hacia el aseguramiento universal en salud en el Perú. Rev Peru Med Exp Salud Publica. 2009;26(2):232-5. doi: 10.17843/ rpmesp.2009.262.1364

21. Berríos CX, Bedregal GP, Guzmán AB. Costo-efectividad de la promoción de la salud en Chile: Experiencia del programa “iMírame!”. Rev Med Chile. 2004;132(3):361-70.

22. Mezones-Holguín E, Díaz-Romero R, Castillo-Jayme J, Jerí-de-Pinho M, Benites-Zapata V, Marquez-Bobadilla E, et al. Promoción de los derechos en salud en Perú: una aproximación desde la perspectiva de acción de la Superintendencia Nacional de Salud. Rev Peru Med Exp Salud Publica. 2016;33(3). doi:10.17843/ rpmesp.2016.333.2302

23. Mezones-Holguin E, Solis-Cóndor R, Benites-Zapata VA, Garnica-Pinazo G, Márquez-Bobadilla E, TantaleánDel-Águila $\mathrm{M}$, et al. Diferencias institucionales en el insuficiente acceso efectivo a medicamentos prescritos en instituciones prestadoras de servicios de salud en Perú: Análisis de la Encuesta Nacional de Satisfacción de Usuarios de los Servicios de Salud (ENSUSALUD 2014). Rev Peru Med Exp Salud Publica. 2016;33(2):205-14. doi: 10.17843/ rpmesp.2016.332.2197

24. Kaufman JS, Mezones-Holguín E. Una epidemiología social para América Latina: una necesidad más allá de la reflexión sobre las inequidades en salud. Rev Peru Med Exp Salud Publica. 2013;30(4):543-6. doi: 10.17843/ rpmesp.2013.304.230

25. Mezones-Holguín E. Las evaluaciones económicas en salud: hacia un acercamiento entre la academia y la política. Rev Peru Med Exp Salud Publica. 2011;28(3):410-3. doi: 10.17843/rpmesp.2011.283.516

Correspondencia: Vicente Benites Zapata Dirección: Av. Velasco Astete 1398 Santiago de Surco. Lima, Perú

Teléfono: (+511)3726150 anexo 5412

Correo electrónico:vbeniteszapata@gmail.com 